\title{
Sistema de adquisición de datos para determinar el grupo de conexión en el transformador trifásico DL1080
}

\section{Data Acquilsition System for Determining Group of Connection in the Phase Transformer DL1080}

\author{
César Augusto Hernández Suárez \\ Ingeniero electrónico, especialista en Interconexión de Redes y Servicios \\ Telemáticos, magíster en Ciencias de la Información y las Comunicaciones, \\ candidato a doctor en Ingeniería de Sistemas y Computación, docente de la \\ Universidad Distrital Francisco José de Caldas, Bogotá, Colombia. \\ cahernandezs@udistrital.edu.co
}

Víctor Alfonso Gómez SAAVEdRa

Ingeniero eléctrico, tecnólogo en electricidad, estudiante de Especialización en Gestión de Proyectos en Ingeniería, auxiliar de laboratorios de la Universidad Distrital Francisco José de Caldas, Bogotá, Colombia.

vagomezs@correo.udistrital.edu.co

\section{Robín Alejandro Peña Lote}

Ingeniero eléctrico, tecnólogo en electricidad, ingeniero eléctrico de Schneider Electric, Bogotá, Colombia.

Contacto: alejandro2905@gmail.com

Fecha de recepción: 5 de agosto de 2013

Clasificación del artículo: investigación

Fecha de aceptación: 3 de marzo de 2014

Financiamiento: Universidad Distrital Francisco José de Caldas

Palabras clave: configuración de devanados, diagrama vectorial, grupo de conexión, microcontrolador, transformador trifásico

Keywords: microcontroller, three phase transformer, vector diagram, vector group, winding configurations

\section{RESUMEN}

Este artículo presenta el diseño y la implementación de un sistema de adquisición de datos para determinar los diferentes grupos de conexión del transformador trifásico DL1080. Este diseño se basa en herramientas computacionales en hardware y software, como Microchip y Labview, 
respectivamente. En consecuencia, el prototipo medidor posee un algoritmo de alto nivel capaz de realizar el análisis vectorial de tensiones en el transformador que establecen la normatividad nacional e internacional. Además, permite un ambiente interactivo con el usuario por medio de herramientas gráficas, como una pantalla de cristal líquido con sus respectivos comandos y el software desarrollado en Labview llamado Índices Horarios. Estas ayudas visuales ilustran diagramas vectoriales, señales de tensión en tiempo real y conexión de devanados, entre otros. La anterior aplicación académica permite integrar una herramienta en el aprendizaje y fortalecimiento de áreas como conversión electromagnética, maquinas eléctricas y sistemas de potencia, entre otras. Por lo tanto, los docentes y los estudiantes podrán reforzar sus conocimientos del área, a través de un elemento didáctico para fomentar un entorno participativo y constructivista.

\section{INTRODUCCIÓN}

En la actualidad, existen analizadores de variables eléctricas para transformadores trifásicos de potencia conocidos como TTR (Fully automated three phase, Transformer Turn Ratio Meter) (Instrument, 2013). Sin embargo, la tecnología actual no cuenta con protocolos de comunicación por radiofrecuencia que garantice distancias de seguridad al momento de realizar pruebas a equipos de potencia como transformadores trifásicos. Por tal razón, en el presente proyecto se desarrolló un prototipo con las mismas cualidades de registro de un TTR en la identificación de los grupos de conexión, además de implementar trasmisión de datos por protocolos ZigBee en un ambiente interactivo y educativo con el estudiante y el docente, algo que resulta innovador en la nueva utilidad de equipos de educación y trabajo en laboratorio.

\section{ABSTRACT}

This paper presents the design and implementation of a data acquisition system to identify the different vector groups of the triphase transformer DL1080. This design is based on computational tools in hardware and software such as Microchips and Labview respectively. Consequently, the prototype has a high-level algorithm. It is able to perform vector analysis of voltage in the transformer which established national and international regulations. This project also allows an interactive environment with the user through graphical tools such as a liquid crystal display with their respective buttons and software developed in Labview called "indices Horarios". This is useful to illustrate: vector diagrams, winding configurations, real time voltage, among others. This academic application is a tool in learning and strengthening areas such as: electromagnetic conversion, electrical machines and power systems. Therefore, teachers and students can reinforce their knowledge.

Para el estudio del grupo de conexión se empleó el transformador trifásico DL1080 de devanados separados, perteneciente al laboratorio de máquinas eléctricas de la Facultad Tecnológica. A continuación se diseñaron los sensores de registro de tensión, con la particularidad de acondicionar las tensiones a niveles admisibles de entrada en el microcontrolador PIC18F4550. Este último es el encargado de realizar un alto procesamiento de datos, concerniente a establecer valores de medida, control sobre los módulos de conmutación, protocolos de comunicación con elementos externos como una pantalla de cristal líquido (LCD gráfica 128X64) y el software Índices Horarios desarrollado en Labview.

De acuerdo con los parámetros de estudio, el prototipo realiza un análisis vectorial de las tensiones entre los devanados primario y secundario y tam- 
bién evalúa el desfase en las formas de onda, para así determinar el respectivo grupo de conexión. Posteriormente, de manera gráfica en la LCD, así como en el software, se visualizan las conexiones de los devanados y asimismo un reloj que ilustra el índice dependiendo el tipo de configuración. En la figura 1 se presenta un diagrama en bloques del diseño del prototipo, en donde se puede apreciar cada uno de los módulos que lo conforman.

Con el diseño y la construcción del prototipo se logró optimizar el tiempo entre prácticas de laboratorio, de manera tal que fue posible realizar 36 diferentes tipos de conexiones del transformador, así como contribuir con el uso de herramientas computacionales de vanguardia para el fortalecimiento de conceptos teórico-prácticos en las asignaturas de tecnología e ingeniería eléctrica.

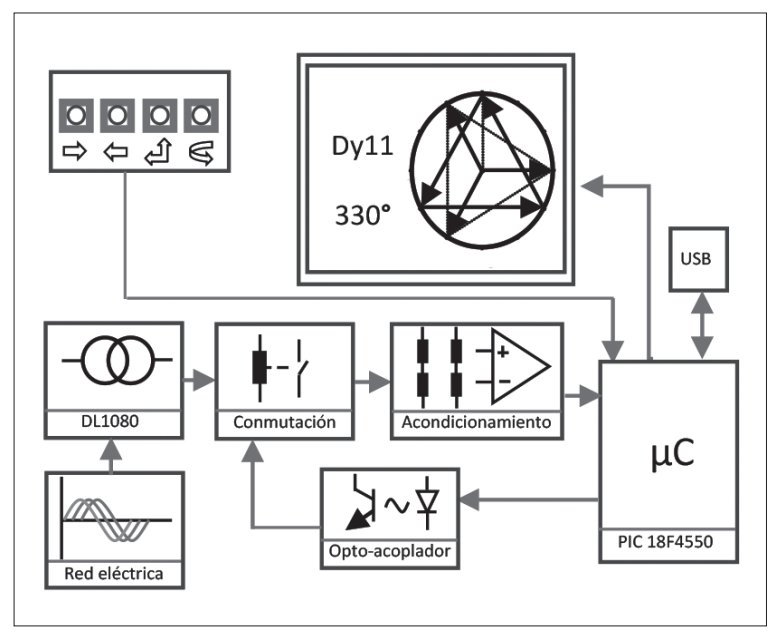

Figura 1. Diagrama bloques prototipo

Fuente: elaboración propia.

\section{GRUPOS DE CONEXIÓN}

En los transformadores trifásicos, según como se realice la conexión de los devanados, se obtienen diferentes relaciones de transformación y diferentes desfases entre las tensiones de entrada y salida. En los circuitos trifásicos estas conexiones son típicamente conocidas como: $D y, Y d, D d, Y y$, $Y z$ y $D z$, tal como lo menciona Vaz Gades (2004). Dichas conexiones satisfacen una aplicación o uso específico.

En los transformadores monofásicos las tensiones del devanado secundario pueden estar en fase o en contrafase, pero en los transformadores trifásicos las tensiones de los devanados secundarios, con respecto a las tensiones de devanados primarias (de fase o de línea), pueden desfasarse en cualquier ángulo múltiplo de $30^{\circ}$ dependiendo de la forma de conexión de los devanados (Staff, 2003).

El índice horario es el ángulo entre la tensión del devanado primario y la tensión de igual nombre del secundario, como se aprecia en la figura 2 entre $V A B$ y $V a b$. Cabe resaltar que este desfase sucede entre las tensiones de fase o de línea. Debido a la simetría de los sistemas trifásicos, se puede deducir que cualquier desfase será siempre múltiplo de $30^{\circ}$ para cualquier conexión que se establezca. Por lo tanto, hay 12 posibles desfases que se pueden asimilar con un reloj, en donde situamos el vector de tensión con mayor magnitud siempre en dirección de las 12 y el vector de menor magnitud en dirección del índice correspondiente. Por ejemplo, un desfase de $330^{\circ}$ corresponde con el índice 11, como se muestra en la figura 2.

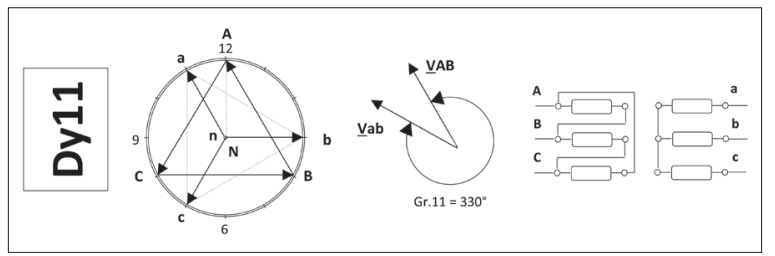

Figura 2. Diagrama de reloj y conexionado del Grupo Dy11

Fuente: elaboración propia. 
El grupo de conexión de un transformador indica el tipo de conexión de los devanados (Delta, Ye o Zeta) y su índice horario. Suele constar de dos letras y un número. La primera letra, siempre en mayúscula, indica la conexión del devanado de alta, en tanto que la segunda, siempre en minúscula, indica la conexión del devanado de baja, y el número es el índice horario (entre 0 y 11).

Es importante hacer notar que los grupos $D y, Y d$ e $Y z$ siempre van a tener un índice horario impar, mientras que los grupos $Y y, D d$ y $D z$ siempre van a tener un índice horario par. Además, debido a que existen diferentes formas de conectar los devanados para conformar cada grupo de conexión - hay dos formas de conectar una $Y$, cuatro formas de conectar una Delta y cuatro formas de conectar una $Z$-, se puede demostrar que en las transformaciones con índice horario impar, variando la forma de conexión del grupo, se obtiene cualquier otro índice impar. Aunque esto también se puede lograr variando exclusivamente sus conexiones de entrada y salida a la red. Pero cuando las transformaciones tienen un índice horario par, variando exclusivamente sus conexiones de entrada y salida a la red tan solo se obtienen tres índices horarios posibles; en un caso " 0,4 y 8 " y en el otro "2, 6 y 10", respectivamente (Lawhead, Hamilton y Horak, 2006). De esta forma, existen unas conexiones denominadas principales y otras denominadas conexiones derivadas, como se ve en la tabla 1. Con lo cual se puede concluir que existen 36 diferentes grupos de conexión.

Tabla 1. Índices horarios en transformaciones trifásicas

\begin{tabular}{|c|c|c|c|c|c|}
\hline Conexiones principales & \multicolumn{5}{|c|}{ Conexiones derivadas } \\
\hline Grupo 0 & 4 & 8 & - & - & - \\
\hline Grupo 1 & 3 & 5 & 7 & 9 & 11 \\
\hline Grupo 2 & 6 & 10 & - & - & - \\
\hline
\end{tabular}

Fuente: elaboración propia.
El índice horario es de suma importancia al conectar transformadores trifásicos en paralelo, ya que dos tensiones que tengan el mismo valor eficaz y diferente ángulo suponen un cortocircuito. Así, por lo tanto, es condición indispensable que dos transformadores conectados en paralelo tengan el mismo grupo de conexión o grupos compatibles entre sí.

\section{NORMATIVIDAD}

La norma NTC - ISO/IEC 17025:2005 establece los requisitos generales para la competencia de laboratorios de ensayo y calibración. En ella se establecen los criterios para los transformadores eléctricos trifásicos cuando se quiere realizar el ensayo de la identificación del grupo de conexión. Por esta razón, se deben tener en cuenta las siguientes normas técnicas:

- IEC 60076-1 (2000), Transformadores de potencia, parte 1: generalidades

- ANSI-IEEE Std. C 57.12.90-2006, Pruebas estándar para transformadores de regulación, potencia y distribución.

- NTC471-1974, Transformadores. Relación de transformación. Verificación de la polaridad y relación de fase.

Estas normas, básicamente, definen los tipos de conexión (Delta, Ye o Zeta), los símbolos, la nomenclatura para cada grupo de conexión y el modelamiento vectorial en el método del reloj. Para el desarrollo de este proyecto se tuvieron en cuenta dichas normas, además del manual de la máquina DeLorenzo DL1080.

\section{Ejecución de la prueba}

El diagrama fasorial de cualquier transformador trifásico que define el desplazamiento angular 


\section{investigación}

puede ser verificado de la siguiente forma (C5712-90, 1999):

- Se conecta el terminal de un devanado con el correspondiente del otro y se excita el transformador. La elección del lado de la alimentación y de la tensión de prueba es totalmente indiferente para el resultado final.

- Se mide la tensión entre varios pares de terminales.

- Se grafican estos valores o se compara la magnitud con la ayuda de una tabla.

La realización del primer paso permite aprovechar al máximo la diferente composición vectorial entre los voltajes de los devanados de alta tensión (AT) y el devanado de baja tensión (BT), según el ángulo de fase entre las dos ternas. En el segundo paso, como se muestra a continuación, solo es necesario tomar cuatro lecturas de tensiones. El último paso requiere la ayuda de una tabla, en donde se comparan las magnitudes de las tensiones medidas y se determina el índice horario del grupo.

El diagrama vectorial de la figura 2 describe una conexión Dy1. Teniendo en cuenta el puente que se hace (terminales "A" de alta y "a" de baja) en el paso uno del ensayo, se tendrá un diagrama como el de la figura 3 en donde se muestran las cuatro tensiones que se van a medir: $V C b, V C c$, $V B c$ y $V B b$.

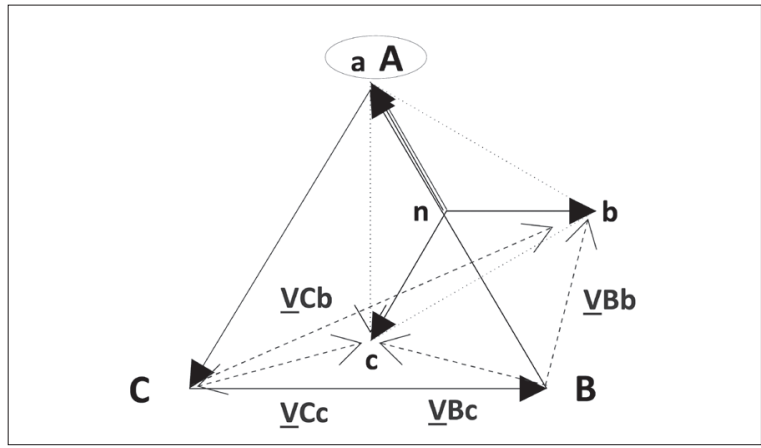

Figura 3. Diagrama vectorial conectando "A con a" Fuente: elaboración propia.

\section{TRANSFORMADOR TRIFÁSICO DL1080}

La máquina DL1080 es un transformador trifásico de devanados separados con una tensión de primario de $380 \mathrm{~V}_{\mathrm{LL}}$ y una tensión de secundario de $170 \mathrm{~V}_{\mathrm{LL}}$; su potencia nominal es de $1 \mathrm{kVA}$. Este elemento del laboratorio de máquinas eléctricas es empleado para prácticas de laboratorio como resistencia de los devanados, relación de transformación, prueba de corto circuito, voltaje en vacío, grupo de conexión. Este último de mayor importancia para el desarrollo del presente proyecto. En la figura 4 se ilustra una imagen de la parte frontal del transformador.

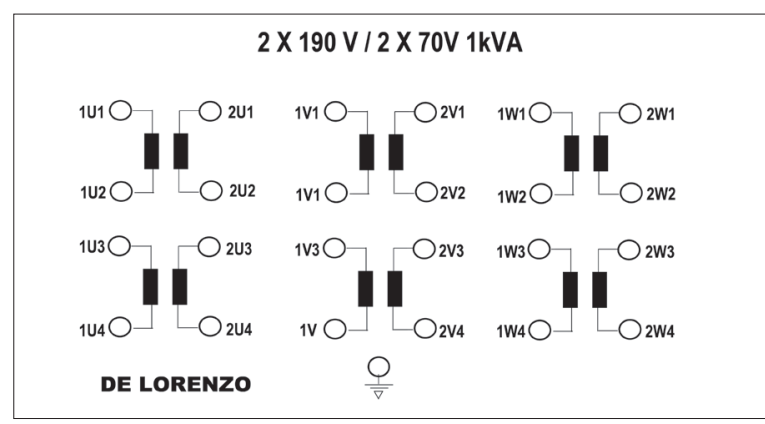

Figura 4. Transformador DL 1080

Fuente: elaboración propia.

\section{METODOLOGÍA}

\section{Diseño e implementación}

El diseño del presente trabajo se hizo con herramientas computacionales tales como Labview, CCS Compiler y Altium Designer. Igualmente, todas las fases del diseño fueron evaluadas en los laboratorios de máquinas eléctricas de la Universidad Distrital Francisco José de Caldas. A continuación se describen las fases de diseño e implementación del prototipo.

\section{Acondicionamiento de tensión}

Las tensiones que se registran tienen magnitudes superiores a los valores máximos de compatibili- 
dad con el elemento que hace la trasformación a la señal digital. Por tal razón, los sensores de voltaje fueron diseñados teniendo en cuenta criterios como: voltaje máximo de entrada en el microcontrolador (5 VDC) y constante de voltaje $(2,5$ VDC) para subir la señal atenuada a valores positivos, tales que el semiciclo negativo de la señal de tensión no afecte el correcto funcionamiento del microcontrolador. En consecuencia, se emplearon dos divisores de voltaje (resistivo puro) para medir la diferencia de tensiones línea-línea y línea-neutro para cada respectiva conexión. En la figura 5 se ilustra un diagrama esquemático del circuito de acondicionamiento.

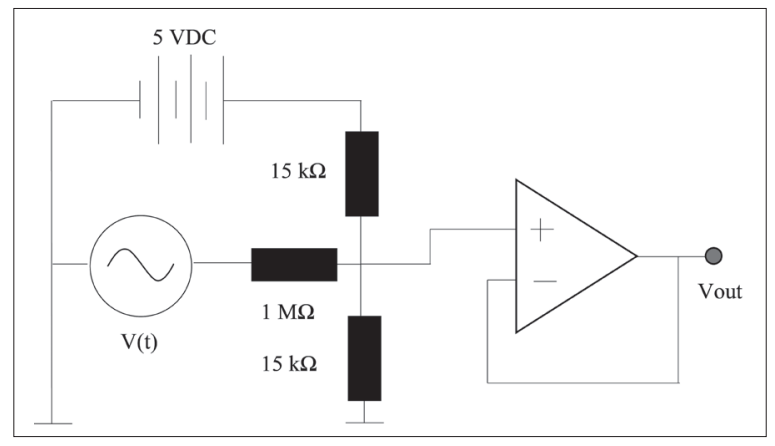

Figura 5. Circuito de acondicionamiento

Fuente: elaboración propia.

La elección de las resistencias R1, R2 y R3 para los dos divisores de medida diferencial obedece a criterios de tensión estacionaria y tensión pico de impulsos transitorio, como lo expone Electrotechnical (2003). Lo anterior a fin de garantizar el nivel de protección del registro de tensión que para el prototipo es CAT III 1000V - CAT IV 600 V. La relación de transformación del sensor se describe en la ecuación (1).

$$
R T=\frac{\mathrm{R} 1}{\mathrm{R} 2 \| \mathrm{R} 3}
$$

En donde:

$\mathrm{R} 1=1 \mathrm{M} \Omega 1 / 4 \mathrm{~W}$ (precisión 2\%)

$\mathrm{R} 2=\mathrm{R} 3=15 \mathrm{k} \Omega \frac{1}{1 / 4} \mathrm{~W}$ (precisión 2\%)
Una sobretensión subamortiguada de segundo orden, debido a capacidades e inductancias parasitas, puede afectar la compatibilidad electromagnética del microcontrolador (Getz, 1996), más aún en el momento en que este realiza el control sobre el módulo de conmutación para la medida diferencial de tensión en los devanados. Por ende, fue necesario adaptar un amplificador operacional en configuración como seguidor, con una tensión de alimentación de 6,3 VDC que regula los valores máximos de entrada, además de generar alta impedancia de entrada en la parte baja de los divisores resistivo para el registro de tensión.

\section{Conmutación de sensor}

El sistema de conmutación diseñado le permite al prototipo cortocircuitar los terminales comunes de AT y BT que especifican las normatividades, además de permitir la lectura de tensiones en forma ordenada para determinar la forma de conexión de los devanados (Delta, Ye o Zeta). Así mismo, determinar el índice horario, por medio de la medición de las cinco tensiones y realizar una medida simultanea de tensiones en alta y baja para verificar el desfase que indica el índice.

Es importante tener presente que para medir tensiones de línea, la forma de entrada del voltaje alterno a los ADC es indiferente. Pero cuando se quieren medir tensiones de fase con respecto a un neutro imaginario (como es el caso de la conexión Delta) existen tensiones flotantes que pueden ser medidas por el prototipo y alterar la determinación del grupo de conexión. Para solucionar esto, las tensiones de fase que mide el prototipo entran con la línea a tierra (o negativo de la fuente) y el neutro al ADC correspondiente. En la tabla 2 se puede ver la función que cumple cada relé dentro del módulo de conmutación. Cabe hacer notar que la conmutación ordenada de los relés la hace directamente el cerebro del prototipo. 
Tabla 2. Función de los relés dentro del módulo de conmutación

\begin{tabular}{|c|c|c|c|}
\hline Relé & Función & ADC Utilizado & Propósito \\
\hline R1 & Puente & - & Primer paso del ensayo \\
\hline R3 - R5 & Medir VCc & 1 & \multirow{4}{*}{ Determinación del índice horario } \\
\hline $\mathrm{R} 3-\mathrm{R} 4$ & Medir VCb & 1 & \\
\hline R2 - R5 & Medir VBc & 0 & \\
\hline $\mathrm{R} 3-\mathrm{R} 4$ & Medir VBb & 0 & \\
\hline $\mathrm{R} 6-\mathrm{R} 7-\mathrm{R} 2-\mathrm{R} 1$ & Medir VAB contra Vab & 1 y 0 & Verificación de desfase \\
\hline $\mathrm{R} 6-\mathrm{R} 2$ & Medir VAB & 0 & \multirow{2}{*}{ Medición de la conexión de alta: $D \circ Y$} \\
\hline $\mathrm{R} 6-\mathrm{R} 8$ & Medir VAN & 1 & \\
\hline $\mathrm{R} 6-\mathrm{R} 1-\mathrm{R} 7$ & Medir Vab & 1 & Medición de la conexión de baja: $d, y o z$ \\
\hline
\end{tabular}

Fuente: elaboración propia.

\section{Procesamiento}

El modulo se compone básicamente de un microcontrolador PIC18F4550 que cuenta con módulos analog-to-digital converter (ADC) que permiten registrar los voltajes alternos (AC) de entrada en el prototipo. Cuenta con una memoria de programa de 32 Kbyte, la cual es ocupada en un $90 \%$ por el algoritmo diseñado y soporta la comunicación USB para enviar datos al computador.

\section{Funciones implementadas}

En la figura 6 se observa el esquema del módulo de procesamiento y análisis en donde se encuentran los ADC, las salidas a los 10 relés, la conexión de la pantalla, los cuatro botones (izquierda, derecha, entrar y salir) y los pines del USB. En este módulo se encuentra implementado un algoritmo en lenguaje $\mathrm{C}$ que permite mostrar un menú principal en donde el usuario puede seleccionar cada función del prototipo:

Realizar prueba: activa los relés del módulo de conmutación, hace un análisis y calcula el voltaje rms de las tensiones entrantes, determina el tipo de conexión de los devanados y calcula el índice horario, para finalmente hallar el grupo de conexión del transformador bajo ensayo.
Resultados: muestra en una tabla las tensiones medidas por el prototipo y el grupo de conexión determinado.

Diagrama vectorial: muestra por pantalla, el grupo de conexión medido, el desfase entre las señales (formas de onda alta y baja tensión) y un diagrama vectorial del grupo de conexión, dentro de un reloj.

Osciloscopio: muestra en tiempo real una gráfica con un voltaje de alta y uno correspondiente de baja, con el fin de evidenciar el desfase entre las señales indicado por el índice horario medido. Para esta función el prototipo conmuta los relés indicados.

Relación de transformación: muestra el grupo de conexión y la relación de transformación compuesta, determinada con los voltajes medidos dentro del ensayo. Esto es importante ya que la relación de transformación cambia con cada grupo de conexión.

Conexión a PC: establece los protocolos de comunicación con el computador y los módulos de radiofrecuencia Zigbee. Posteriormente, envía los datos medidos por el prototipo dentro del ensayo. 


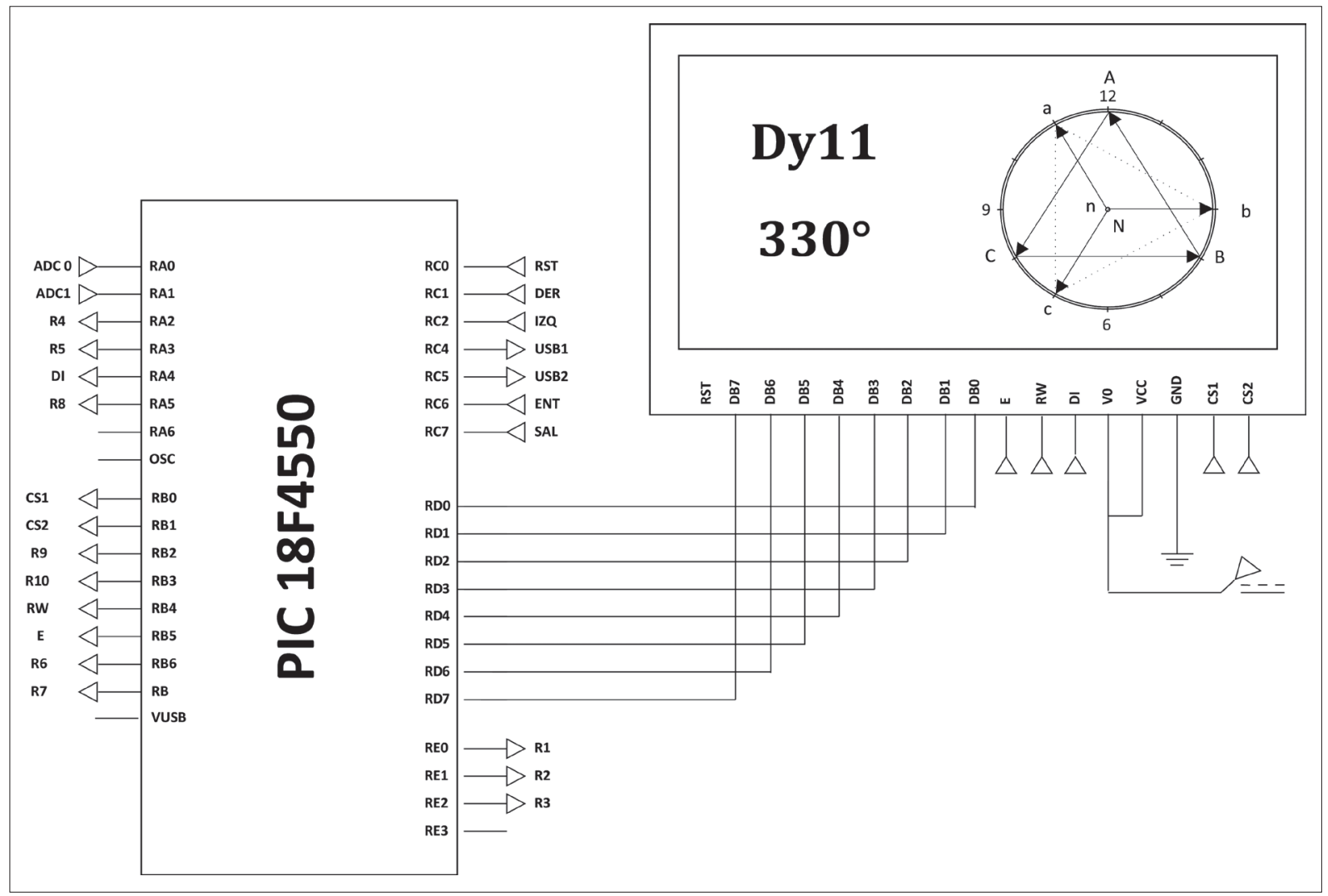

Figura 6. Procesamiento y registro

Fuente: elaboración propia.

Tratamiento de la señal

Teniendo en cuenta los voltajes rms medidos por el prototipo, que a su vez han presentado la secuencia de registro como lo expresa la tabla 2. Entonces, la determinación de la conexión de los devanados se hace de la siguiente forma:

- Para el devanado de alta: se comparan las tensiones VAB y VAN. Si VAN $=\mathrm{VAB} / \sqrt{3}$, el prototipo determina una conexión $Y e$. De forma contraria, determina una conexión Delta.

- Para el devanado de baja: se comparan las tensiones $\mathrm{Vab}$ y $\mathrm{Vbn}$. Si $\mathrm{Vbn}=\mathrm{Vab} / \sqrt{ } 3$, el prototipo determina una conexión $Y e$. De forma contraria, determina una conexión Delta. Cuando se determina una conexión
$Y e$, si $\mathrm{Vzn}=\mathrm{Vab} / 3$, el prototipo determina una conexión Zeta.

A la hora de realizar el ensayo, es importante hacer las conexiones del prototipo al transformador de los neutros de alta, baja y la conexión del terminal z (si estos existen según el tipo de conexión de los devanados).

Para la determinación del índice horario, el cerebro del prototipo analiza las cinco tensiones indicadas en la tabla 2 y por medio de una comparación de estas deduce el grupo de conexión.

El algoritmo diseñado permite determinar el valor eficaz de la forma de onda de tensión, a partir de la definición que establece la ecuación (2). En tal sentido, el algoritmo establece el procesamiento 
de datos de manera discreta y mediante integración numérica por Simpson.

$$
V_{r m s}=\sqrt{\frac{1}{T} \int_{0}^{T} v(t)^{2} d t}
$$

\section{Módulo de interfaz con el usuario}

Está compuesto por una pantalla LCD Gráfica (module with back light) HDM64GS12 de 1024 pixeles. Además, cuenta con cuatro pulsadores con las funciones: derecha, izquierda, entrar y salir.

\section{Comunicación USB (Universal Serial Bus)}

Para el presente proyecto se evaluaron los diferentes protocolos de comunicación, como por ejemplo el USB CDC (Communication Device Class), el cual emula un puerto serie COM para el direccionamiento de datos. Sin embargo, en la etapa de implementación, la comunicación resultó ser muy lenta para la transferencia de información. Seguidamente, se implementó el protocolo de comunicación USB Bulk Transfer o USB desencadenado, el cual tiene la particularidad de realizar una comunicación bidireccional masiva entre el ordenador y el prototipo a velocidades de transferencia entre los $60 \mathrm{MB} / \mathrm{s}$ a $15,6 \mathrm{MB} / \mathrm{s}$ (Don, 2001). No obstante, otra gran ventaja fue el lograr establecer la comunicación en cualquier puerto del ordenador, sin excepción alguna, mediante los VID (Vendor ID) \&PID (Product ID) de identificación entre microchip y Microsoft, así como también el controlador de dispositivo o driver mchpusb.sys proporcionado por microchip para la identificación del prototipo en sistemas operativos Windows 7 de 64 bits.

\section{Recepción de señales DAQ}

Una vez tomados los valores de tensión por el microcontrolador PIC18F4550 para su posterior digitalización, son enviados mediante comunicación ZigBee a un dispositivo esclavo, que en este caso se denominó "sonda de comunicación". Esta tiene conectividad USB con el ordenador. Allí el algoritmo grafico de programación compara los valores de entrada para asignar macros de control en la ejecución de subrutinas para gráficos de conexión devanados del transformador, magnitudes de tensiones registradas, modo scope e índice horario monitoreado.

Para garantizar una efectiva medición del grupo de conexión, se desarrolló en Labview el procesamiento digital concerniente a la obtención de los coeficientes de Fourier, con base en los datos registrados por el microcontrolador. Lo anterior, a fin de obtener la componente fundamental de tensión $(60 \mathrm{~Hz})$ y de esta manera observar si existe un error en la medida del grupo de conexión cuando se realiza la lectura energizando el transformador con la señal de la red eléctrica, cuya distorsión armónica es de aproximadamente el $4,2 \%$. No obstante, este criterio de diseño se estableció por los armónicos que se pueden presentar por las corrientes de magnetización en el transformador, aun cuando este no presente carga en el devanado secundario (Candela García, 2009). La cuantización y el tratamiento de la señal se expresan mediante la ecuación (3).

\section{Software Índices Horarios}

$$
f(t)=a_{0}+\sum_{n=1}^{N} a_{n} \cos n w_{o} t+\sum_{n=1}^{N} b_{n} \operatorname{sen} n w_{o} t
$$

Los avances tecnológicos en software y hardware facilitan un mejor entendimiento de los problemas a nivel de ingeniería, ya que permiten dimensionar y establecer parámetros indicativos para así poder llevarlos a un plano didáctico y comprensible para las personas (Chilan Santos y Mera Quimi, 2009). Por tal razón, en el presente trabajo se desarrolló un software en la plataforma de programación gráfica Labview para generar un 
entorno más amigable para estudiantes y docentes en el aprendizaje de los grupos de conexión del transformador DL1080. En la figura 7 se ilustra el software con el nombre Índices Horarios.

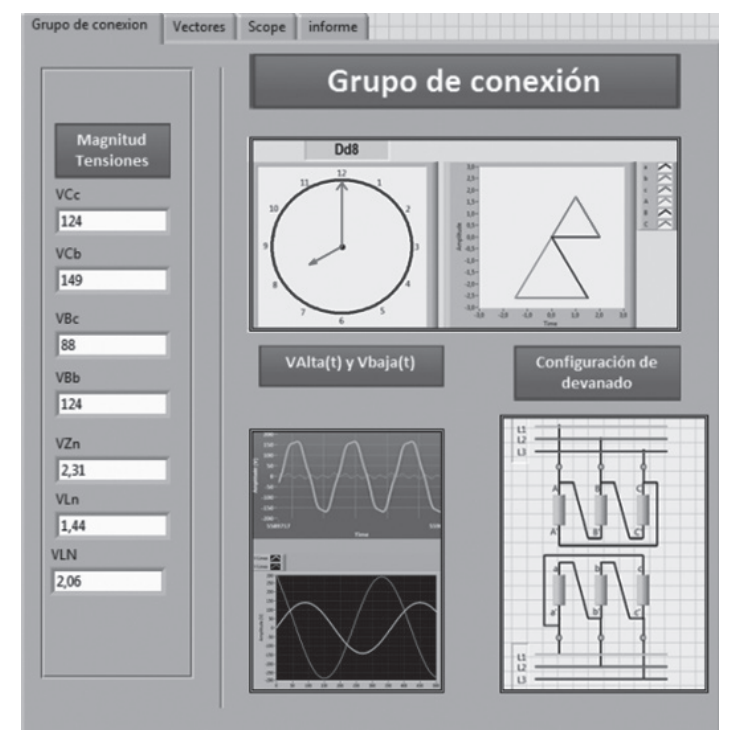

Figura 7. Software Índices Horarios

Fuente: elaboración propia

\section{PRUEBAS Y RESULTADOS}

A continuación se exponen las diferentes pruebas que validan el correcto funcionamiento del prototipo para su implementación y uso en las prácticas de laboratorio concernientes a grupos de conexiones en la maquina DL1080.

\section{Circuito de acondicionamiento}

Para esta prueba se inyectaron tensiones dentro y fuera del rango de medición del sensor de voltaje, con lo cual se obtuvieron señales con y sin saturación. Nótese en la figura 8 que al aplicar la tensión nominal del prototipo (220 Vrms), el máximo valor de tensión en la resistencia de baja corresponde a 5 Vrms. De igual manera, al aplicar una tensión de $440 \mathrm{Vrms}$, el sensor presenta saturación en la resistencia de baja. Sin embargo, no se supera el valor máximo de entrada en el microcontrolador 5 Vrms. Con lo anterior, el prototipo cumple la normatividad IEC 61010 en la categoría de protección en la medida de tensión en régimen estacionario.
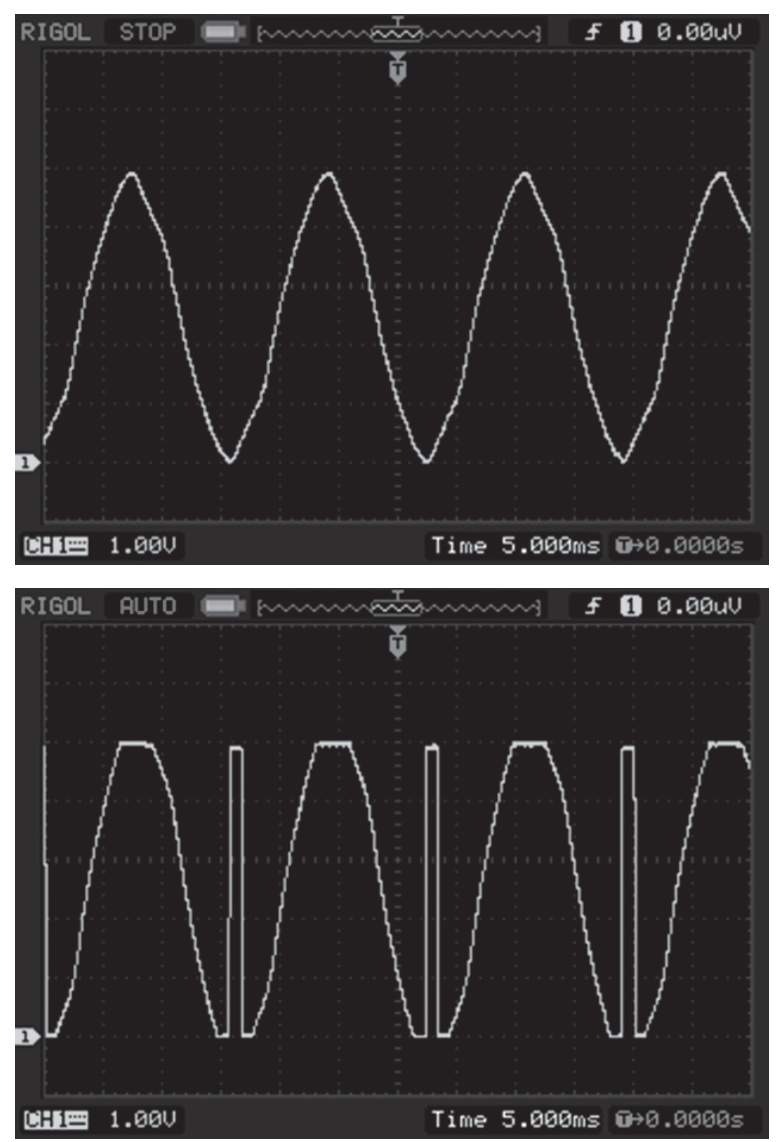

Figura 8. Tensión de baja

Fuente: elaboración propia.

\section{Lectura de voltaje rms}

En esta prueba se hizo un barrido de tensión desde cero hasta 440 Vrms, para comprobar con respecto al diseño los límites de voltaje que registra el prototipo y la precisión en la medida. De esta manera, se llevó una fuente AC a $440 \mathrm{Vrms}$ y se encontró que el prototipo mide de forma correcta 


\section{investigación}

hasta los 220 Vrms. Después de este voltaje el seguidor del módulo de acondicionamiento recorta la señal de entrada como opción de protección. Por lo tanto, se comprobó que el rango de medida del prototipo está entre 0 y 220 Vrms. Dentro de esta misma prueba se encontró que el prototipo mide con una incertidumbre de $\pm 1,0 \mathrm{~V}$.

\section{Determinación del grupo de conexión}

En el manual de usuario de la maquina DL1080 se encuentra una tabla que contempla todos los grupos de conexión. Sin embargo, las relaciones de tensiones $(V C b, V C c, V B c$ y $V B b)$ para los índices horarios 2, 3 y 4 y los índices 8,9 y 10 son iguales. Por lo que existe el problema al tratar de determinar grupos de conexión con índices horarios pares, debido a que no se distinguiría entre los índices 2, 4 y 8, 10 (ya que al momento de comparar las tensiones las relaciones entre ellas son las mismas). Por ejemplo, no se podría distinguir entre un grupo $Y y 2$ y un grupo $Y y 4$ o entre un grupo $D z 8$ y un $D d 10$, entre otros.

Haciendo un análisis del diagrama vectorial de estos grupos, se determinó que al medir la tensión $V A B$ y comprobándola con la tensión ya medida $V B b$ para los índices 2 y 4 , y con $V C c$ para los índices 8 y 10, se podía distinguir entre todos los índices horarios pares.

Una vez diseñadas las condiciones de análisis vectorial de tensiones, se procede a desarrollar los 36 diferentes grupos de conexión sobre el transformador DL1080. De tal forma, se registraron todos los grupos y como resultado el prototipo midió de forma correcta las configuraciones de los devanados de AT y BT, además de todos los índices horarios. Igualmente, el prototipo graficó en la pantalla los diagramas vectoriales y los desfases entre las señales en la función osciloscopio.

\section{Prueba de comunicación USB}

Una vez desarrollados los 36 tipos de conexiones en el transformador, estos se enviaron al ordenador, con lo cual se obtuvieron los diferentes índices horarios, señales de tensión, diagramas vectoriales y configuración de los devanados.

Los datos enviados al ordenador fueron direccionados a un prototipo esclavo o sonda de comunicación, a través de módulos de radio frecuencia $\mathrm{XBee}$, en una red punto a punto configurada con los microcontroladores. De esta manera, se garantizaron distancias de seguridad en las pruebas de determinación del índice horario en el transformador trifásico.

\section{CONCLUSIONES}

El prototipo está en capacidad de identificar el grupo de conexión aun cuando el transformador se encuentra conectado a la red eléctrica y esta presenta una distorsión armónica en la señal de tensión del 4,2\%. No obstante, las corrientes de magnetización de la máquina DL1080 en sus diferentes configuraciones no repercuten en un error en la determinación de su índice horario.

Se obtuvo una precisión del $2 \%$, con una resolución de $0,01 \mathrm{~V}$ en la medición de voltaje rms, por lo que la determinación del grupo de conexión se hace de forma correcta en todos los casos. El rango de voltaje que mide el prototipo es de 0 a 220 VAC. Las anteriores especificaciones son idóneas para la realización de los ensayos, ya que el valor de voltaje y el lado de la alimentación (alta o baja) dentro de la prueba son indiferentes con los resultados.

El prototipo registra de forma correcta todos los 36 grupos de conexiones contemplados dentro 
del proyecto (Dd - Dy - Yd - Dy - Dz -Yz). De igual manera, el usuario puede verificar por medio del prototipo y de la conectividad inalámbrica los índices horarios que interpreta el software desarrollado en Labview. Por ejemplo: el diagrama vectorial, el desfase entre las señales de los devanados de alta y baja tensión y las tensiones medidas en la determinación de cada grupo.

Este proyecto ha cumplido con su objetivo general, es decir, se ha diseñado y construido un sistema de adquisición de datos para determinar el grupo de conexión en el transformador trifásico DL1080. Esta herramienta de aprendizaje será de gran ayuda para docentes y estudiantes en el desarrollo de prácticas de laboratorio.

\section{FINANCIAMIENTO}

Este artículo se deriva del proyecto de investigación Sistema de adquisición de datos para determinar el grupo de conexión en el transformador trifásico DL1080, el cual fue financiado por la Universidad Distrital Francisco José de Caldas.

\section{RECOMENDACIONES Y TRABAJOS FUTUROS}

Para determinar grupos de conexión en transformadores de alta potencia, solo se debe realizar un circuito amplificador del voltaje en el devanado de baja y adaptarlo al módulo de acondicionamiento, de forma tal que se puedan registrar tensiones dentro de la resolución de adquisición de señales del prototipo.

\section{REFERENCIAS}

C57-12-90, I. S. IEEE Standard Test Code for Liquid-Immersed Distribution, Power, and Regulating Transformers, Estados Unidos: The Institute of Electrical and Electronics Engineers, 1999.

Candela García, J. I., Aportaciones al filtrado hibrido paralelo en redes trifásicas de cuatro hilos (sistemas eléctricos de energía renovable) (tesis doctoral), Universidad Politecnica de Catalunya, Barcelona, 2009.

Chilan Santos, B. y Mera Quimi, R. E., Adquisición, graficación y procesamiento de señales de los motores y transformadores del laboratorio de maquinaria eléctrica de la fiec basado en la plataforma de programación labview, Guayaquil: Escuela Superior Politecnica del Litoral, 2009.
Dharita, P., J, C., Ashish, P., \& N., M. "A User Friendly Simulation for Transformer Vector Group", En 2012 International Conference on Communication Systems and Network Technologies, Rajkot, Gujarat, India: IEEEXplore, 2012.

Don, A., USB System Architecture, Massachusetts: MindShare, 2001.

Electrotechnical, C. I. International Standar IEC 61010, Safety Requirements for Electrical Equipment for Measurement, Control, and Laboratory Use, Ginebra: Rue de Varembé, 2003.

Getz, R., Understanding and Eliminating EMI in Microcontroller Applications, Dallas, Texas, Estados Unidos: National Semiconductor Corporation, 1996. 


\section{investigación}

Instrument, T., Fully Automated Three Phase Transformer Turns Ratio Meter, Hipotronics, 2013.

Lawhead, L., Hamilton, R. y Horak, J., Three Phase Transformer Winding Configurations and Differential Relay Compensation, Georgia, Estados Unidos: Basler Electric Company, 2006.
Staff, E. E., Circuitos magnéticos y transformadores, Barcelona: Reverté, 2003.

Vaz Gades, M., Transformadores ligaçoes e esfasamientos, Porto, Portugal: Universidad do Porto, Facultade de Engenharía, 2004. 